\title{
Agonistic sensory effects of airborne chemicals in mixtures: Odor, nasal pungency, and eye irritation
}

\author{
J. ENRIQUE COMETTO-MUÑIZ and WILLIAM S. CAIN \\ University of California, San Diego, La Jolla, Califormia \\ and \\ H. KENNETH HUDNELL \\ U. S. Environmental Protection Agency, Research Triangle Park, North Carolina
}

\begin{abstract}
Threshold responses of odor, nasal pungency (irritation), and eye irritation were measured for single chemicals (1-propanol, 1-hexanol, ethyl acetate, heptyl acetate, 2-pentanone, 2-heptanone, toluene, ethyl benzene, and propyl benzene) and mixtures of them (two three-component mixtures, two sixcomponent mixtures, and one nine-component mixture). Nasal pungency was measured in subjects lacking a functional sense of smell (i.e., anosmics) to avoid interference from olfaction. Various degrees of stimulus agonism (additive effects) were observed for each of the three sensory channels when testing mixtures. As the number of components and the lipophilicity of such components in the mixtures increased, so did the degree of agonism. Synergistic stimulus agonism characterized the eyeirritation response for the most complex (the nine-component) and the most lipophilic (one of the sixcomponent) mixtures. Physicochemical properties play a large role in the determination of sensitivity to airborne chemicals, particularly to their ability to evoke irritation. While this has revealed itself previously with respect to single chemicals, it seems to have relevance to mixtures as well.
\end{abstract}

Airborne chemicals in the environment are detected by humans through two sensory channels: olfaction and the so-called common chemical sense (CCS), or chemesthesia. The sense of smell is mediated by the olfactory nerve (cranial nerve 1), whereas the facial CCS (from the various mucosae: ocular, nasal, and oral) is principally mediated by the trigeminal nerve (cranial nerve $V$ ).

Since almost any odorant-at a high enough concentration-evokes not only odor but also chemesthetic sensations, an issue in human chemoreception entails distinguishing between a purely olfactory and a combined olfactory-trigeminal nasal response. We have begun to clarify this matter by measuring nasal detection thresholds to airborne compounds in subjects with a normal

The research was supported by research Grants 5 RO1 DC 0028412 and 1 R29 DC 02741-01 from the National Institute on Deafness and Other Communication Disorders, and by the U. S. Environmental Protection Agency through Cooperative Agreement CR-816362-02. It has been reviewed by the Agency and cleared for publication, but does not necessarily reflect the views of the Agency and no official endorsement should be inferred. Mention of trade names or commercial products does not constitute endorsement or recommendation for use. Thanks are extended to Robin Babbitt and Todd O'Hearn for their excellent technical assistance. A version of this paper was presented by W.S.C. at the "Healthy Buildings ' 95 " conference in Milan, Italy, and was included in the Proceedings. The research was performed while J.E.C.-M. was a member of the Carrera del Investigador Cientifico, Consejo Nacional de Investigaciones Cientificas y Técnicas (CONICET), República Argentina. Correspondence should be directed to J. E. Cometto-Muñiz, Chemosensory Perception Laboratory, Department of Surgery (Otolaryngology), Mail Code 0957, University of California, San Diego, La Jolla, CA 92093-0957 (e-mail: ecometto@ ucsdedu). sense of smell (i.e., normosmics) and comparing them with those obtained in subjects without a sense of smell (i.e., anosmics) (Cometto-Muñiz \& Cain, 1990, 1991, $1993,1994)$. When a substance becomes barely detectable to a normosmic, we have assumed this to correspond to the odor threshold. When a substance becomes barely detectable to an anosmic, we have assumed this to correspond to the nasal pungency threshold. An interest in a broad spectrum of sensory responses from airborne chemicals has led us also to measure eye irritation thresholds (Cometto-Muñiz \& Cain, 1991, 1995b).

In our previous studies, the stimuli comprised homologous series of volatile organic compounds (VOCs), for example, alcohols, acetates, ketones, and alkylbenzenes. Our aim was to relate physicochemical properties - which change systematically in such series-with their sensory potency. The outcome showed that potency increases (i.e., thresholds decrease) with increasing carbon chain length. The relationship is similar, but not identical, for the olfactory and chemesthetic modalities. Ultimately, potency gets to a point where there is no further increase or there is even a decrease. Nasal pungency and eye-irritation thresholds occurred at similar concentrations, whereas odor thresholds occurred one or more orders of magnitude below. Across chemical series, the chemesthetic thresholds held a uniform relationship with a simple physicochemical property (viz., saturated vapor concentration). Odor thresholds failed to conform to a uniform relationship across series with any such individual property, as far as we could discern.

In the present investigation, we continued our strategy of resorting to anosmics to uncover "odor-unbiased" nasal 
(a)

$$
\begin{aligned}
& 0.000 \text { INTG } \#=8 \\
& 5.500 \text { ATT } 2^{\wedge}=1 \\
& 5.500 \text { THRSH }=0 \\
& 6.750 \text { ATT } 2^{\wedge}=6 \\
& 7.700 \text { ATT } 2^{\wedge}=3
\end{aligned}
$$

PEAK CAPACITY:

ZERO $=20,0.396$

$\mathrm{ATT} 2^{\wedge}=-1$

CHT SP $=1.0$

AR REJ $=0$

THRSH $=-1$

PK WD $=0.06$

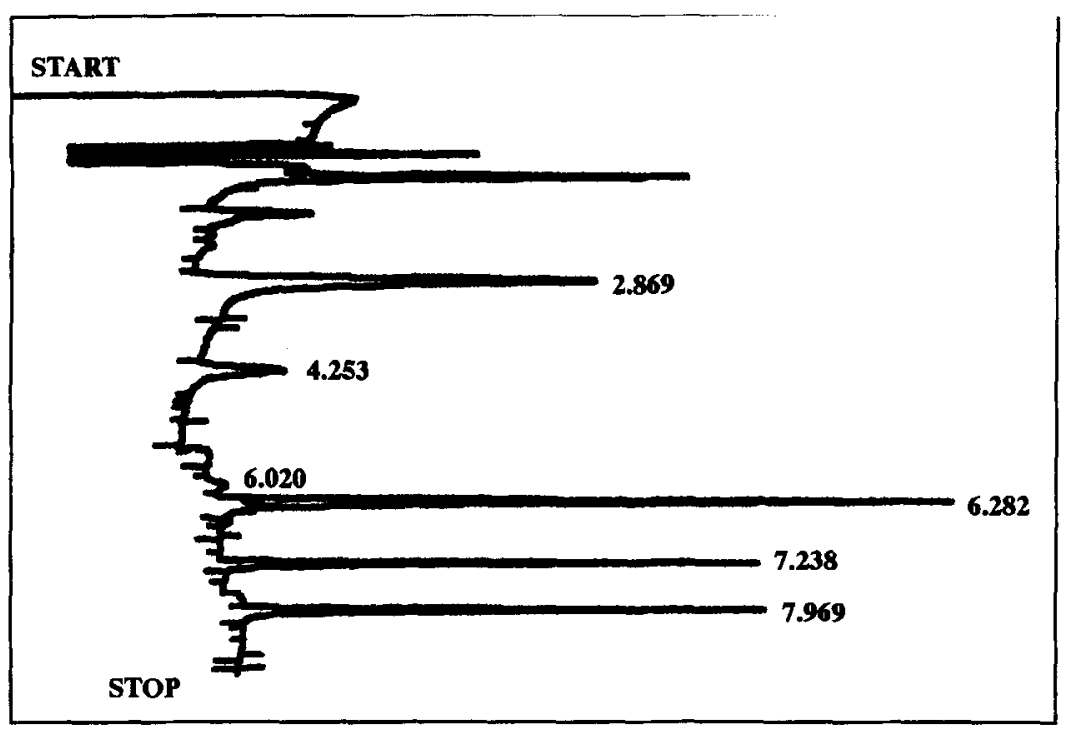

\begin{tabular}{r|r|l|l|r} 
RT & AREA & TYPE & WIDTH & AREA \% \\
\hline $\mathbf{2 . 8 6 9}$ & $\mathbf{1 5 4 7 4}$ & PB & $\mathbf{. 1 5 4}$ & 1.18534 \\
$\mathbf{4 . 2 5 3}$ & $\mathbf{3 2 5 7}$ & BP & $\mathbf{. 1 4 7}$ & .24949 \\
$\mathbf{6 . 0 2 0}$ & $\mathbf{1 3 4 6}$ & PP & .093 & .10311 \\
$\mathbf{6 . 2 8 2}$ & $\mathbf{5 4 0 4 4}$ & PB & $\mathbf{. 0 7 3}$ & 4.13988 \\
$\mathbf{7 . 2 3 8}$ & $\mathbf{8 4 7 5 6 9}$ & PB & $\mathbf{. 0 4 9}$ & 64.92544 \\
$\mathbf{7 . 9 6 9}$ & $\mathbf{1 2 2 5 2 1}$ & VB & $\mathbf{. 0 5 8}$ & 9.38534 \\
\hline
\end{tabular}

TOTAL AREA $=1305450$

MUL FACTOR $=1.0000 \mathrm{E}+00$

Figure 1a Sample chromatogram from Mixture $C$ at Dilution Step 6. From start to end, the numbers represent the retention times for 1-propanol, ethyl acetate, 1-butanol, 2-pentanone, toluene, and ethyl benzene, respectively. The very earty peaks appear even in "blank" squeezebottles containing odorless mineral oil or deionized water.

pungency thresholds, as well as measuring odor and eyeirritation thresholds, but using mixtures of substances as stimuli. Research in the field of odor mixtures has heavily tilted toward the suprathreshold range. The typical outcome shows that the perceived odor intensity of a mixture falls below the sum of the perceived odor intensities of its separate components (hypoadditivity) (e.g., Berglund \& Olsson, 1993). This result holds even in models considering "addition" of concentration (mass) and not simply addition of sensation (Cain, Schiet, Olsson, \& de Wijk, 1995). Experiments on binary mixtures of pungent odorants at concentrations clearly appealing to the CCS revealed a concentration-dependent degree of addition in the total perceived intensity (ComettoMuñiz, García-Medina, \& Calviño, 1989). A follow-up study showed that the intensity of odor of those same mixtures was always hypoadditive, whereas their intensity of nasal pungency was additive or even hyperadditive (Cometto-Muñiz \& Hernández, 1990).

Studies of the chemosensory perception of mixtures at the threshold level have been scarce and restricted to the olfactory modality. Their outcome suggests simple 
(b)
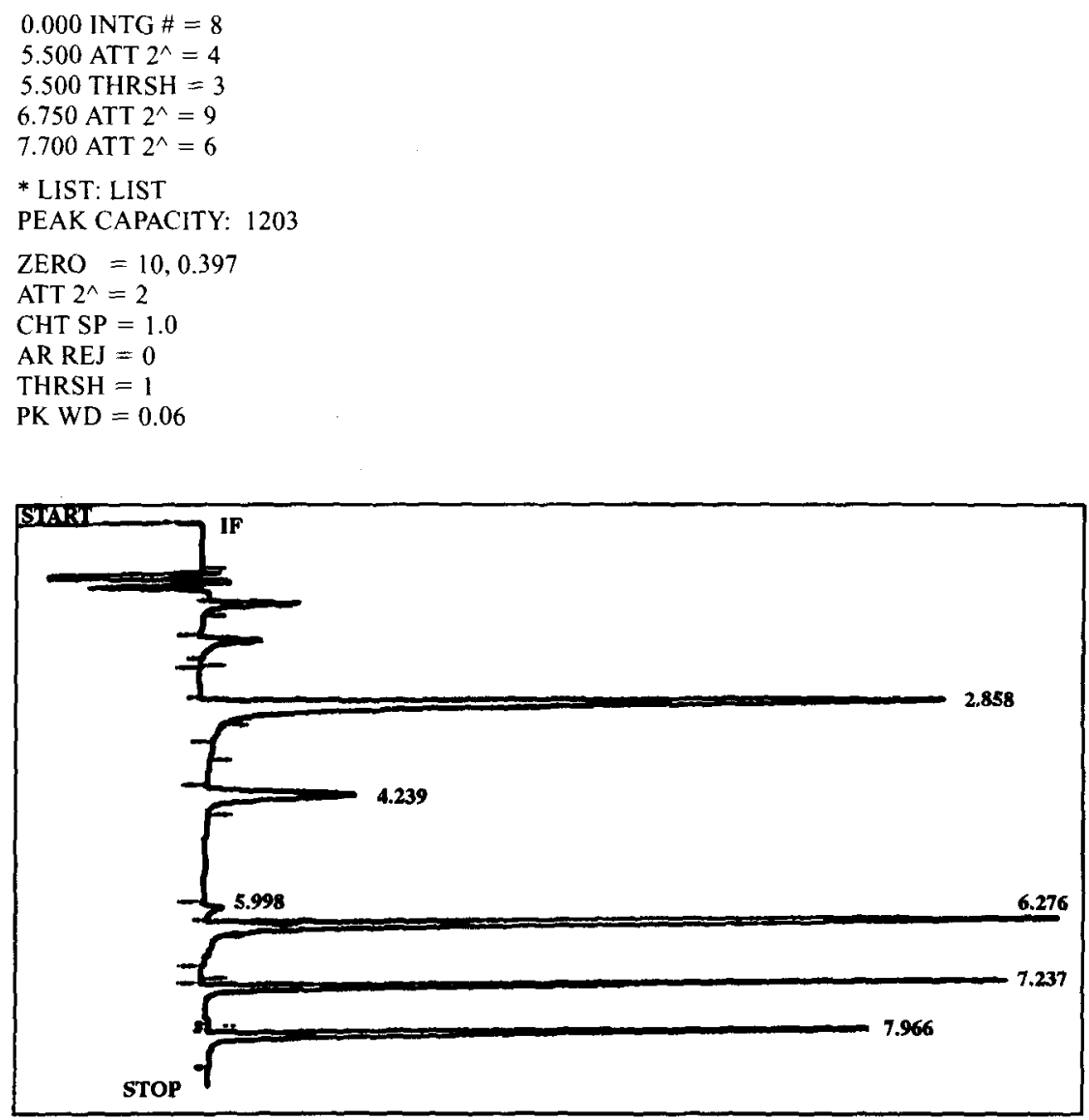

\begin{tabular}{|c|c|c|c|c|}
\hline RT & AREA & TYPE & WIDTH & AREA $\%$ \\
\hline 2.858 & 171953 & PB & .116 & 139194 \\
\hline 4.239 & 40550 & $\mathrm{BB}$ & .135 & 32825 \\
\hline 5.998 & 14169 & $\mathrm{PP}$ & .090 & .11470 \\
\hline 6.276 & 618038 & $\mathrm{~PB}$ & .068 & 5.00294 \\
\hline 7.237 & 10101832 & ISBH & .048 & 81.77302 \\
\hline 7.966 & 1218158 & TVV & .057 & 9.86083 \\
\hline
\end{tabular}

TOTAL AREA $=1.2354 \mathrm{E}+07$

MUL FACTOR $=1.0000 \mathrm{E}+00$

Figure 1b. Sample chromatogram from Mixture $C$ at Dilution Step 4. From start to end, the numbers represent the retention times for 1-propanol, ethyl acetate, 1-butanol, 2-pentanone, toluene, and ethyl benzene, respectively. The very early peaks appear even in "blank" squeezebottles containing odorless mineral oil or deionized water.

stimulus agonism (Guadagni, Buttery, Okano, \& Burr, 1963; Patterson, Stevens, Cain, \& Cometto-Muñiz, 1993 ) with some cases of synergistic stimulus agonism as number of components increased (Baker, 1963; Laska \& Hudson, 1991; Rosen, Peter, \& Middleton, 1962). Simple stimulus agonism in, for example, a balanced 3component mixture implies that when the substances are presented mixed, each needs to be at only one third of its individual threshold concentration for the mixture to be perceived. Synergistic agonism implies-following the same example of a three-component mixture-that individual substances need to be at a concentration even lower than one third of their respective thresholds for the mixture to be perceived, whereas partial agonism implies that they need to be at a concentration higher than one third of their respective thresholds but less than the threshold value itself. Independence implies that at least one of the components in the mixture needs to be at its individual threshold for the mixture to be perceived. Finally, antagonism implies that the components need to 
be at concentrations higher than their individual thresholds for the mixture to be perceived. ${ }^{1}$

In the present experiment, we measured three sensory thresholds: odor, nasal pungency, and eye irritation for individual VOCs and for five mixtures containing three, six, or nine components. In view of the cited research on odor thresholds for chemical mixtures and in view of the success of a quantitative structure-activity relationship that we developed to predict nasal pungency (Abraham, Andonian-Haftvan, Cometto-Muñiz, \& Cain, 1996), we expected that both olfaction and the nasal CCS would show simple agonism with some possibility of an increase in agonism toward synergy as the mixtures increased in number of components. In view of our findings that eye-irritation thresholds equaled nasal pungency thresholds (Cometto-Muñiz \& Cain, 1995b), we anticipated similar behavior in the two channels regarding mixtures, despite differences between the two facial mucosae regarding distribution of fiber types and perireceptor environment (Silver, 1992; Unger, 1992).

\section{METHOD}

\section{Stimuli}

The stimuli included members of homologous series of alcohols (1-propanol, 1-butanol, 1-hexanol), esters (ethyl acetate, hexyl acetate, heptyl acetate), ketones (2-pentanone, 2-heptanone), and alkylbenzenes (toluene, ethyl benzene, and propyl benzene). All were analytical-grade reagents. Single chemicals tested for the three relevant sensory responses of odor, nasal pungency, and eye irritation were: 1-propanol, 1-hexanol, ethyl acetate, heptyl acetate, 2-pentanone, 2-heptanone, toluene, ethyl benzene, and propyl benzene. Mineral oil served as solvent to prepare threefold dilution steps of the pure $(100 \% \mathrm{v} / \mathrm{v})$ substance (i.e., $33 \%, 11 \%, 3.7 \%$, $1.1 \%$, etc., v/v). Due to the limited solubility of 1 -propanol in mineral oil, the first two members of its series, $33 \%$ and $11 \% \mathrm{v} / \mathrm{v}$, were prepared in deionized water.

Five mixtures were prepared using mineral oil as solvent: two three-component mixtures (labeled A and B, below), two sixcomponent mixtures (labeled $C$ and $D$ ), and one nine-component mixture (labeled E). Mixture A comprised l-propanol, ethyl acetate, and 2-pentanone; Mixture B, 1-hexanol, heptyl acetate, and 2heptanone; Mixture C, 1-propanol, 1-butanol, ethyl acetate, 2pentanone, toluene, and ethyl benzene; Mixture D, 1-hexanol, 1heptanol, hexyl acetate, heptyl acetate, 2-heptanone, and propyl benzene; and Mixture E, 1-propanol, 1-hexanol, ethyl acetate, heptyl acetate, 2-pentanone, 2-heptanone, toluene, ethyl benzene, and propyl benzene. These particular mixtures were chosen to give one three-component (i.e., A) and one six-component (i.e., C) mixture of relatively low-molecular-weight, high-vapor-pressure chemicals; one three-component (i.e., B) and one six-component (i.e., D) mixture of higher molecular weight, lower vapor-pressure chemicals; and one nine-component mixture (i.e., E) having both kinds of chemicals.

Substances in each liquid mixture were present in proportions that reflected their odor thresholds measured in previous studies (Cometto-Muñiz \& Cain, 1990, 1991, 1993, 1994). This was done by using a "reference" that contained, for each liquid mixture, components at their individual odor thresholds as found previously. For example, the odor thresholds for 1-propanol, ethyl acetate, and 2-pentanone equaled $0.0051 \%, 0.0017 \%$, and $0.0017 \% \mathrm{v} / \mathrm{v}$, respectively, and the reference for this three-component mixture therefore had these concentrations. On the basis of such a refer- ence, threefold steps in concentration were prepared for each mixture both above and below the reference. Accordingly, the first step for Mixture A above the reference was $0.015 \%(0.0051 \times 3)$, $0.0051 \%(0.0017 \times 3)$, and $0.0051 \%(0.0017 \times 3) \mathrm{v} / \mathrm{v}$, respectively, for 1-propanol, ethyl acetate, and 2-pentanone. The first step below the reference was $0.0017 \%(0.0051 \div 3), 0.00056 \%$ $(0.0017 \div 3)$, and $0.00056 \%(0.0017 \div 3) \mathrm{v} / \mathrm{v}$, respectively, for the same chemicals in the same order. Steps above the reference continued until they reached the maximum value that could be presented in a mixture of the specified proportions (e.g., for Mixture A, 33\%, $11 \%$, and $11 \% \mathrm{v} / \mathrm{v}$ of 1 -propanol, ethyl acetate, and 2-pentanone, respectively). Steps below the threshold continued until they fell definitively below the odor threshold for even the most sensitive subject.

We prepared duplicate series for each of the nine single chemicals and each of the five mixtures. Stimuli were delivered from cylindrical, squeezable, high-density polyethylene bottles $(270-\mathrm{ml}$ capacity) (Cain, 1989) containing $30 \mathrm{ml}$ of solution. For measurements of odor and nasal pungency, the bottle closure had a pop-up spout that fitted into the nostril being tested. Each nostril was tested separately. For measurements of eye irritation, the bottle caps held a tube that led to a $25-\mathrm{ml}$ measuring chamber (of the type used in variable-volume dispensers), the rim of which was placed around the eye. Each eye was tested separately. The tube that fed the chamber was connected to the headspace of the bottle. A squeeze of the bottle delivered a puff of vapor into the measuring chamber where the eye was exposed. A polyethylene dust cover closed the open end of the measuring chamber when the bottle was not in use.

\section{Gas Chromatography}

The concentration of each compound in the headspace of every bottle was measured by a gas chromatograph (photoionization detector) equipped with a gas-sampling valve, allowing direct sampling of the headspace. For every single or mixed stimulus, repeated chromatographic readings were taken at each dilution step. Readings were also taken from bottles containing pure chemicals $(100 \% \mathrm{v} / \mathrm{v})$. The headspace of a bottle with a pure chemical contains vapor saturated with the chemical at room temperature $\left(23^{\circ} \mathrm{C}\right)$. The concentration of the saturated vapor from each substance was retrieved from the literature. Knowledge of saturated vapor concentration (at $23^{\circ} \mathrm{C}$ ) and its associated average chromatographic reading allowed conversion of the readings from the other bottles into concentration units (parts per million by volume).

Figures $1 \mathrm{a}$ and $\mathrm{lb}$ show two sample chromatograms from Mixture C at Dilution Steps 6 and 4, respectively. All stimuli were run on a column DB- $1,30 \mathrm{~m} \times 0.53 \mathrm{~mm}$ i.d. used alone or in series with a DB-WAX, $30 \mathrm{~m} \times 0.53 \mathrm{~mm}$ i.d. Both columns were obtained from J\&W Scientific, Folsom, CA. As illustrated by these typical chromatograms, degree of resolution of the components in mixtures was good. The selection of relatively unreactive chemicals and the use of members on opposite ends of homologous chemical series contributed to make the chromatographic resolution of the components if not easy at least achievable.

Figure 2 presents, as an example, the concentrations derived from gas chromatographic readings for the dilution series of Mixture $\mathrm{C}$ obtained for each of its six components. Vapor-phase concentration of any compound at a given liquid dilution step was approximately the same whether the compound was alone or in a three-, six-, or nine-component mixture. Using the data for ethyl acetate, Figure 3a illustrates a case in which there was almost perfect agreement in the vapor-phase concentration of a fixed liquid dilution irrespective of whether the target chemical was alone or mixed with others. Using the data for 2-pentanone, Figure $3 \mathrm{~b}$ illustrates a case where there was close but imperfect agreement. Even with this trend toward almost perfect agreement, thresholds were converted from liquid dilution steps into vapor-phase con- 


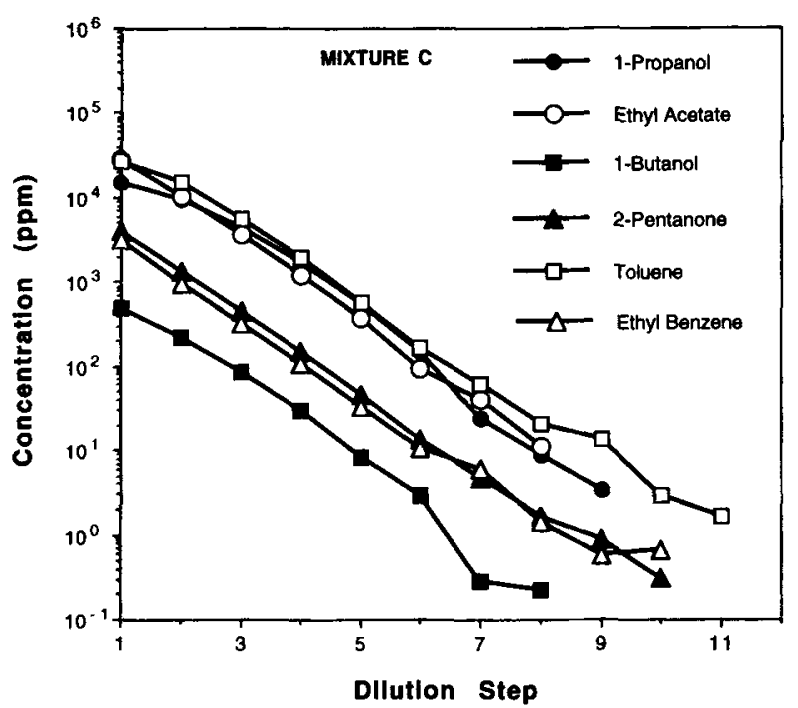

Figure 2. Vapor-phase concentration (parts per million), derived from chromatographic readings, as a function of liquid dilution step for the components in Mixture $C$.

centrations via the actual curves that corresponded to the particular circumstances being considered, single stimuli or mixed stimuli.

\section{Subjects}

Eight subjects, 4 males and 4 females, provided values for odor and eye-irritation thresholds. The subjects covered a wide range of age ( 21 to 60 years) in order to match the group of anosmics available for testing nasal pungency thresholds. Participants included a male and a female in each of the following categories: early $20 \mathrm{~s}$, early $30 \mathrm{~s}$, early $40 \mathrm{~s}$, and late $50 \mathrm{~s} / 60$. A 21 -year-old male became unavailable after being tested for odor thresholds. To complete the group, he was replaced by a 22 -year-old male tested only for eyeirritation thresholds.

Four clinically diagnosed anosmic subjects, 2 males and 2 females, provided values for nasal pungency thresholds. One participant was a head-trauma anosmic (male, 66 years old), the other 3 were congenital anosmics (a male, 43 years old, and 2 females, 20 and 62 years old).

\section{Procedure}

On odor and nasal pungency trials, participants delivered the stimulus by inserting the pop-out probe inside the specified nostril and squeezing the bottle as they sniffed. On eye-irritation trials, they squeezed the bottle while the eye was exposed in the measuring chamber. The subjects quickly learned to squeeze and sniff with equal vigor on every trial.

A forced-choice ascending method of limits served to assess threshold. The subject had to choose the stronger smelling or stronger feeling of two stimuli. One was a blank of mineral oil and, at the start, the other was a high dilution-step, low concentration of the stimulus, either single chemical or mixture. If the choice was correct, testing continued with the same step from the duplicate set, also paired with a blank. If the choice was incorrect, testing continued with the next step-a liquid-phase concentration three times higher-paired with a blank. In this way, correct choices entailed the presentation of the same concentration; errors triggered step-wise increments in concentration. The procedure continued until five correct choices were made in a row, in which case that step was taken as threshold. Once the threshold was measured for one nostril or eye, the other nostril or eye was tested. After this, testing began again with another stimulus. Both the ascendingconcentration approach to the threshold and the separate testing of each nostril helped to minimize the effects of adaptation that are frequently encountered in olfactory investigations (ComettoMuñiz \& Cain, 1995a).

Sessions typically lasted between 1 and $2 \mathrm{~h}$ and were repeated until eight thresholds per subject, four for each nostril or eye, were obtained for each single compound and each mixture. This equaled a total of 64 odor or eye-irritation thresholds per stimulus and 32 nasal pungency thresholds per stimulus.

\section{Data Analysis}

Geometric means served to summarize threshold concentrations within and among subjects. The geometric mean acknowledges that threshold data for chemosensory stimuli conform to log nor-

(a)

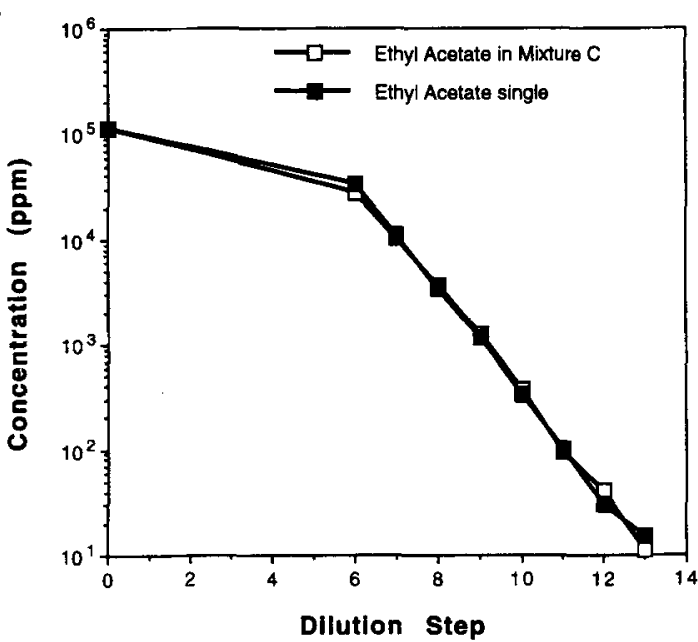

(b)

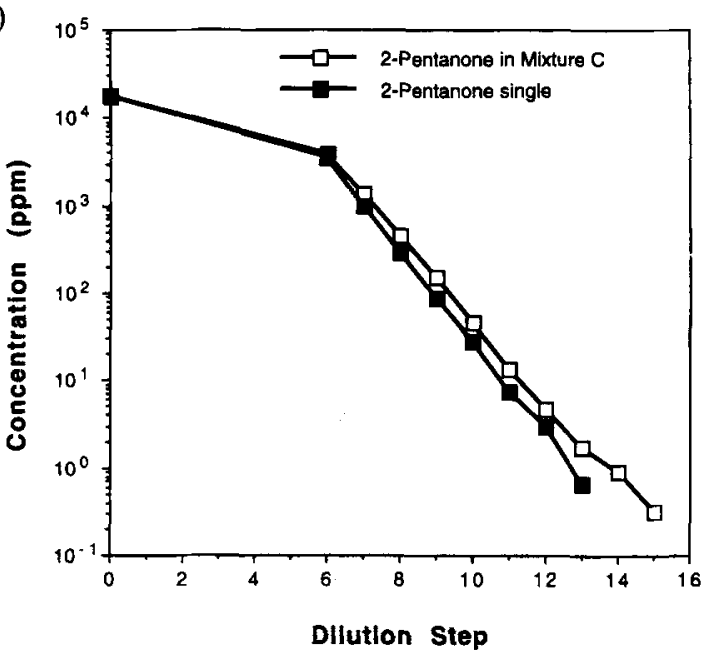

Figure 3. (a) Vapor-phase concentration of ethyl acetate derived from chromatographic readings as a function of liquid dilution step when this chemical was single or part of Mixture C. Dilution Step 0 represents undiluted ethyl acetate $(100 \% \mathrm{v} / \mathrm{v})$. Dilution Step 6 in this plot corresponds to Dilution Step 1 in the ethyl acetate function of Figure 2. (b) Idem for 2-pentanone. Dilution Step 0 represents undiluted 2-pentanone, and Dilution Step 6 in this plot corresponds to Dilution Step 1 in the 2-pentanone function of Figure 2. 
mal distributions (Amoore, 1986; Brown, MacLean, \& Robinette, 1968; Cain \& Gent, 1991).

For analysis of additivity in mixtures, consider the case of a three-component mixture with a formulation that reflects perfectly a subject's relative sensitivity to its three components unmixed. Such a mixture would be perfectly balanced. If the components were simply to add their individual effects, detection would occur when each component fell at one third the concentration of its unmixed threshold. Hence, if component $A$ had a threshold of $3 \mathrm{ppm}$, component $\mathrm{B}$ of $9 \mathrm{ppm}$, and component $\mathrm{C}$ of $27 \mathrm{ppm}$, and if the mixture were made up in the proportions 1:3:9, respectively, for balance, we would expect detection for the mixture to occur with component $\mathrm{A}$ at $1 \mathrm{ppm}$, component $\mathrm{B}$ at $3 \mathrm{ppm}$, and component $\mathrm{C}$ at $9 \mathrm{ppm}$.

The relative contribution of components in a just detectable, 3 component mixture can be reflected in the formula: WS = $a\left(R_{\mathrm{A}}\right)+b\left(R_{\mathrm{B}}\right)+c\left(R_{\mathrm{C}}\right)$, where WS stands for weighted sum; $R_{\mathrm{A}}=$ concentration of $\mathrm{A}$ in mixture $\div$ threshold $\mathrm{A}, R_{\mathrm{B}}=$ concentration of $\mathrm{B}$ in mixture $\div$ threshold $\mathrm{B} ; R_{\mathrm{C}}=$ concentration of $\mathrm{C}$ in mixture $\div$ threshold $\mathrm{C}$, and with $a, b$, and $c$ as weighting coefficients to reflect the degree of balance in the mixture. These weighting coefficients in our three-component mixture are defined as follows: $a=\left[R_{\mathrm{A}} /\left(R_{\mathrm{A}}+R_{\mathrm{B}}+R_{\mathrm{C}}\right)\right] * 3 ; b=\left[R_{\mathrm{B}} /\left(R_{\mathrm{A}}+R_{\mathrm{B}}+R_{\mathrm{C}}\right)\right] * 3 ; c=$ $\left[R_{\mathrm{C}} /\left(R_{\mathrm{A}}+R_{\mathrm{B}}+R_{\mathrm{C}}\right)\right] * 3$. The second factor of each coefficient represents the number of components in the mixture (three in our example).

In our numerical example, WS $=1(1 / 3)+1(1 / 3)+1(1 / 3)=1$, which implies simple agonism of stimulating power. In an unbalanced mixture, which occurs more commonly than not, since no single subject has exactly the same sensitivity as the reference group used to formulate the mixture, $a, b$, and $c$ will take on different values but will always add up to 3 for a three-component mixture, to 6 for a six-component mixture, and to 9 for a ninecomponent mixture. Irrespective of the relative weights of the components, whenever WS lies at 1.0 , simple agonism will have held. Whenever WS lies significantly above or below 1.0, departure from simple agonism will have held. WS above 1.0 implies that the components do not add their sensory potency completely when mixed (partial agonism or even antagonism). WS below 1.0 implies that the components have gained sensory potency when mixed (synergistic agonism).

\section{RESULTS}

Figure 4 presents thresholds (parts per million by volume) for all three sensory responses plotted for each of nine substances. The graph is divided into nine sections corresponding to the substances. Within each section, the first value corresponds to the threshold (odor, eye irritation, and nasal pungency) of the substance when presented alone. Subsequent thresholds correspond to the concentration at which the substance was present when that particular mixture reached threshold (e.g., 1-propanol in $A$ corresponds to the level of 1propanol found in Mixture A when the mixture achieved threshold). A common trend in the three sensory responses and all chemicals is for thresholds to decline with increasing number of components in the mixture. This indicates that some degree of stimulus agonism takes place in the mixtures to help precipitate odor, eye irritation, and nasal pungency when each component falls below its individual sensory threshold. Nevertheless, there are cases in which, when the mixture achieves odor threshold, one of the components approaches its in-

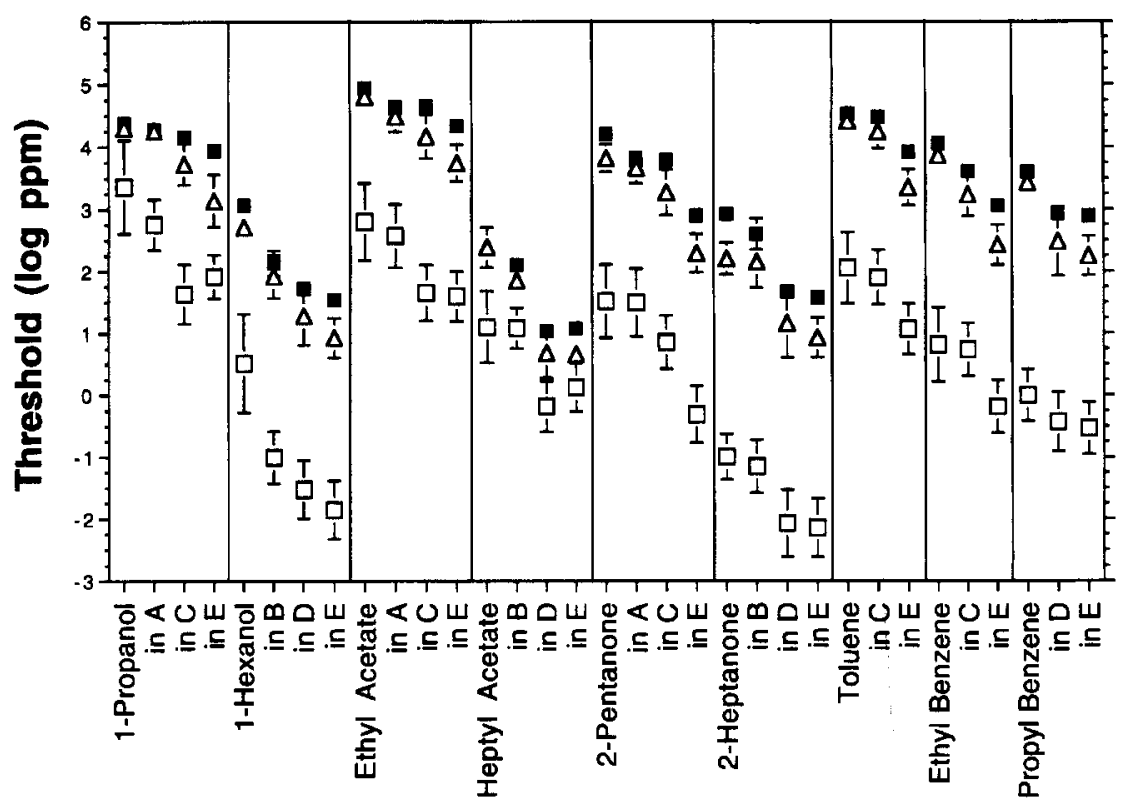

Figure 4. Thresholds ( $\log p p m \pm S D$ ) for nasal pungency (filled squares), eye irritation (triangles), and odor (empty squares). The $S D$ is indicated by bars. The nine sections of the graph correspond to nine substances. Each section lists, first, the threshold for the substance by itself (e.g., 1-propanol), and then, consecutively, the level at which that substance was present when the threshold was achieved for mixtures of increasing complexity-for example, 1-propanol in Mixture A (three components) when $A$ achieved threshold, 1-propanol in $C$ (six components) when $C$ achieved threshold, and 1-propanol in $E$ (nine components) when $\mathrm{E}$ achieved threshold. 
dividual odor threshold (suggesting independence rather than agonism). This holds, particularly, for heptyl acetate in Mixture B and 2-pentanone in Mixture A. We realize that we have certainly not exhausted the issue here; we have just taken-we believe-a thoughtful and careful first step. Considerably more data need to be collected before a broad generalization can be made.

It should be pointed out that pungency thresholds for heptyl acetate and propyl benzene presented alone could be measured in only two to four of the eight repetitions (depending on the anosmic person tested). Probably as a result of this, the pungency threshold for Mixture D could be measured in only two to five of the eight repetitions. Hence, values given for the pungency of these two chemicals and of Mixture D do not represent the average of all anosmics on all repetitions, but do represent the average of the cases in which a threshold was obtained. Also, to limit the already high number of sensory and analytical measurements but still be able to make the contrast between the less lipophilic and more lipophilic substances, we included one compound in Mixture C (1-butanol) and two in Mixture D (1-heptanol and hexyl acetate) whose thresholds in the single state were not measured for the present group of subjects but taken from our previously published data.

Table 1 shows, subject by subject, values of WS from the formula given under Data Analysis above. For averages across subjects, 12 of 15 cases yielded WSs above 1.0 in a way that implies a general tendency for components to act as partial agonists in mixtures. This varied,

Table 1

Weighted Sum of Ratios of Mixed Thresholds/Unmixed Thresholds

\begin{tabular}{|c|c|c|c|c|c|}
\hline \multirow[b]{2}{*}{ Subject } & \multicolumn{2}{|c|}{ Three Components } & \multicolumn{2}{|c|}{ Six Components } & \multirow{2}{*}{$\begin{array}{c}\text { Nine } \\
\text { Components: } \\
\text { Mixture E }\end{array}$} \\
\hline & Mixture A & A Mixture B & Mixture $\mathrm{C}$ & Mixture D & \\
\hline \multicolumn{6}{|c|}{ Odor } \\
\hline 1 & 1.0 & 6.6 & 0.9 & 1.8 & 1.4 \\
\hline 2 & 4.9 & 2.8 & 6.3 & 1.6 & 1.3 \\
\hline 3 & 6.4 & 1.9 & 3.7 & 0.4 & 1.2 \\
\hline 4 & 3.7 & 3.0 & 2.3 & 3.2 & 2.0 \\
\hline 5 & 2.6 & 6.3 & 6.5 & 1.4 & 1.8 \\
\hline 6 & 5.9 & 1.4 & 2.0 & 0.6 & 1.0 \\
\hline 7 & 5.0 & 0.3 & 7.2 & 2.3 & 2.0 \\
\hline 8 & 2.8 & 4.7 & 4.2 & 1.1 & 3.0 \\
\hline Geom. Mean & 3.6 & 2.5 & 3.4 & 1.3 & 1.6 \\
\hline \multicolumn{6}{|c|}{ Eye Irritation } \\
\hline 1 & 2.2 & 6.0 & 3.1 & 0.6 & 0.8 \\
\hline 2 & 1.6 & 0.9 & 2.0 & 0.4 & 0.5 \\
\hline 3 & 1.9 & 1.4 & 3.0 & 0.2 & 0.6 \\
\hline 4 & 1.9 & 1.6 & 2.6 & 0.6 & 0.6 \\
\hline 5 & 1.0 & 0.6 & 2.9 & 0.4 & 0.7 \\
\hline 6 & 2.1 & 1.1 & 1.4 & 0.03 & 0.3 \\
\hline 7 & 3.3 & 2.2 & 2.6 & 0.2 & 0.9 \\
\hline 8 & 2.0 & 4.6 & 1.1 & 0.1 & 0.3 \\
\hline Geom. Mean & 1.9 & 1.7 & 2.2 & 0.2 & 0.6 \\
\hline \multicolumn{6}{|c|}{ Nasal Pungency } \\
\hline I & 2.1 & 0.7 & 5.8 & 0.8 & 1.7 \\
\hline 2 & 2.2 & 2.3 & 7.0 & 0.5 & 1.5 \\
\hline 3 & 1.3 & 2.0 & 6.6 & 1.0 & 1.5 \\
\hline 4 & 2.2 & 0.9 & 6.7 & 1.1 & 1.8 \\
\hline Geom. Mean & 1.9 & 1.3 & 6.5 & 0.8 & 1.6 \\
\hline
\end{tabular}

however, with both sense modality and complexity of a mixture .

An analysis of variance (ANOVA) was performed on the logarithm of the values shown in Table 1 for odor and eye irritation, with the variables of modality (two levels: odor and eye irritation) and mixtures (five levels), given that the same subjects (except 1 ) provided these two sensory thresholds. The results revealed a significant difference between sensory modalities $[F(1,7)=25.86$, $p=.001]$, with eye irritation showing higher stimulus agonism than odor; a significant difference among mixtures $[F(4,28)=14.01, p<.00005]$, with the mixtures with the higher number of components and the more lipophilic components showing greater stimulus agonism; and a significant interaction of sensory modality $\times$ mixture $[F(4,28)=4.37, p=.007]$, which reflected the tendency for complexity to have more leverage for eye irritation.

An alternative ANOVA on the same log values was again performed for odor and eye-irritation data but excluding Mixture $\mathrm{E}$, which contained substances of both low and high lipophilicity, in order to look at the factors of sensory modality, number of components in the mixture, and lipophilicity. The results again revealed significant differences for sensory modality $[F(1,7)=18.98$, $p=.003]$, with eye irritation showing higher stimulus agonism; for number of components $[F(1,7)=9.38, p=$ $.02]$, with the higher number of components showing greater stimulus agonism; and for lipophilicity $[F(1,7)=$ $11.18, p=.01]$, with the mixtures made of the more lipophilic substances showing greater stimulus agonism. The two-way interaction of number of components $x$ lipophilicity was significant $[F(1,7)=23.97, p=.002]$, reflecting that the increase in agonism with the higher number of components was driven by the lipophilic mixtures. Finally, the three-way interaction was also significant $[F(1,7)=8.71, p=.02]$, reflecting a differential effect of lipophilicity and number of components on eye irritation and odor, with the chemesthetic modality showing more agonism.

Two ANOVAs were also performed on analogous data (i.e., $\log$ WS) for nasal pungency. The first was a oneway ANOVA that showed significant differences among mixtures $[F(4,12)=18.40, p<.00005]$, with the mixtures with a higher number of components and more lipophilic components showing greater stimulus agonism. The second was a two-way ANOVA - that, again, excluded Mixture E-showing significant differences for lipophilicity $[F(1,3)=50.26, p=.006]$, with the mixtures made of the more lipophilic substances showing greater stimulus agonism. Also, although the factor of number of components was not significant, the interaction of number of components $\times$ lipophilicity $[F(1,3)=$ $12.10, p=.04]$ was significant, reflecting the gain in agonism with the higher number of components seen in the more lipophilic mixtures but not in the less lipophilic ones.

Confidence intervals $(95 \%)$ allowed us to determine whether the value of $\log$ WS for each mixture and sensory modality differed significantly from 0 (simple ago- 
Table 2

Results of $95 \%$ Confidence Intervals on Log WS: Odor, Eye Irritation, and Nasal Pungency

\begin{tabular}{|c|c|c|c|c|}
\hline Mixture & $N$ & $M$ & $S D$ & Result \\
\hline \multicolumn{5}{|c|}{ Odor } \\
\hline A & 8 & 0.551 & 0.263 & Partial agonism \\
\hline $\mathrm{B}$ & 8 & 0.390 & 0.436 & Partial agonism \\
\hline $\mathrm{C}$ & 8 & 0.532 & 0.317 & Partial agonism \\
\hline D & 8 & 0.115 & 0.296 & Agonism \\
\hline$E$ & 8 & 0.207 & 0.151 & Partial agonism \\
\hline \multicolumn{5}{|c|}{ Eye Irritation } \\
\hline A & 8 & 0.281 & 0.145 & Partial agonism \\
\hline $\mathrm{B}$ & 8 & 0.238 & 0.343 & Agonism \\
\hline $\mathrm{C}$ & 8 & 0.344 & 0.167 & Partial agonism \\
\hline $\mathrm{D}$ & 8 & -0.645 & 0.444 & Synergistic agonism \\
\hline $\mathrm{E}$ & 8 & -0.261 & 0.180 & Synergistic agonism \\
\hline \multicolumn{5}{|c|}{ Nasal Pungency } \\
\hline $\mathrm{A}$ & 4 & 0.285 & 0.124 & Partial agonism \\
\hline $\mathrm{B}$ & 4 & 0.113 & 0.246 & Agonism \\
\hline $\mathrm{c}$ & 4 & 0.813 & 0.035 & Partial agonism \\
\hline D & 4 & -0.093 & 0.157 & Agonism \\
\hline $\mathrm{E}$ & 4 & 0.203 & 0.046 & Partial agonism \\
\hline
\end{tabular}

nism), that is, if WS differed significantly from 1 . The results are presented in Table 2. Again, the general picture shows that mixtures increase their degree of stimulus agonism with the increasing number of components and the increasing lipophilicity of such components. Eye irritation even reached synergistic agonism for the most lipophilic (D) and the most complex (E) mixtures.

\section{DISCUSSION}

A study of odor thresholds for 1-butanol, $p$-cresol, pyridine, and their various two- and three-component mixtures at different proportions found simple agonism most commonly (Rosen et al., 1962). However, the threecomponent mixture at two proportions showed significant synergistic agonism.

In an investigation of odor thresholds for substances associated with food aromas, Guadagni et al. (1963) found an approximation to simple agonism among components in all but 1 of 20 mixtures studied. The mixtures had between 2 and 10 components, and included a number of saturated and unsaturated aliphatic aldehydes, an alcohol, a carboxylic acid, a sulfide, and an amine.

Baker (1963) studied odor thresholds for eight chemicals, for their binary mixtures, and for the eightcomponent mixture. Unlike the previous studies, this investigation found synergism for many of the pairs and one case of antagonism for the pair $m$-cresol-acetophenone. The eight-component mixture showed synergism.

More recently, Laska and Hudson (1991) measured odor-detection thresholds for various single chemicals and for three-component mixtures. The general outcome suggested some degree of agonism, including possible synergistic agonism for a six-component mixture. The latter was based on estimated concentrations of the single components of the mixture at threshold.
None of the studies up to and including Laska and Hudson included calibration of the vapor phase of the mixtures. As Figure $2 b$ revealed, estimation of concentration could lead to error and the relatively small departure seen with the present mixtures cannot be generalized to other mixtures. A detailed investigation of one ternary mixture composed of 1-butanol, 2-pentanone, and $n$-butyl acetate, and of its three components, included gas chromatography of the headspace of all stimuli (Patterson et al., 1993). Furthermore, the mixture was formulated to be equisensorial on the basis of measured average odor thresholds for the individual componentsthresholds obtained in recent previous studies by a similar procedure in the same laboratory. The outcome showed simple stimulus agonism: when the ternary mixture achieved odor threshold, the headspace concentration of each component was approximately one third that of the component's individual odor threshold.

Compared with the broad spectrum of individual odorants, the present study included a relatively similar fraction, which might seem likely to promote agonism. As indicated, previous studies have also shown agonism (though without calibration of headspace), and some even more than the present study, with stimuli that equaled or exceeded the present set in diversity. Based on the present outcome for calibrated stimuli, there remains no doubt that agonism holds and perhaps does so more frequently than not. Agonism may indeed cut across all chemical boundaries. The data highlight the need for further study into factors that control degree of agonism. At this stage, it is unclear whether a generic answer or stimulus-specific answers will hold. The present research has focused more on general rules than on stimulusspecific answers, which must properly begin with binary mixtures.

The degree of agonism in mixtures at threshold depends on the possibility for different molecules to share some aspect of the process of stimulation, though not necessarily common receptors. One way to look at the matter would be to classify molecules according to some common features in the stimulation process and then test thresholds for mixtures of compounds within and across classification groups. Despite the discovery of two secondmessenger pathways - the cyclic AMP and the inositol triphosphate pathways (see review in Anholt, 1993) - use of this apparent dichotomy to explore psychophysically meaningful differences in functioning has yet to prove successful. Thresholds for mixtures provide another domain for such exploration.

In the case of nasal irritation, the use of the compound capsaicin (trans-8-methyl- $N$-vanillyl-6-nonenamide; present in red peppers) could provide a basis for a classification of airborne irritants. Experimental work in animals has shown that local and systemic pretreatment with capsaicin produces insensitivity to subsequent challenges with chemical irritants (Lundblad, Lundberg, \& Änggård, 1984; Silver, Farley, \& Finger, 1991; Silver, Mason, Marshall, \& Maruniak, 1985). So far, the evidence suggests that capsaicin acts on certain $\mathrm{C}$ fibers, 
particularly polymodal nociceptors, and some $\mathrm{A}_{\delta}$ fibers (Buck \& Burks, 1986). Topical application of capsaicin in the human nasal mucosa produces sneezing, secretion, and a burning and painful sensation that can be prevented with a local anesthetic (Geppetti et al., 1988; Stjärne, Lundblad, Lundberg, \& Änggård, 1989). Repetitive local application in the human nasal mucosa also produces desensitization (Sicuteri et al., 1989). The development of a safe and quantitative method to deliver different capsaicin concentrations to the nose of humans would allow testing its sensitizing and desensitizing properties in the nose-as done in the mouth (Green, 1993) - and the extent to which the response to airborne pungent chemicals is diminished, eliminated, or unaffected. This could yield information on the generality of the capsaicinbased desensitization of the nasal cavity and, perhaps, a basis for the classification of airborne irritants. A classification done in such a way could provide a frame to guide selection of mixtures to be tested for degree of agonism to trigger sensory irritation.

The present investigation suggests that lipophilicity enhances the chemosensory potency of mixtures, and this has implications regarding the solvent properties of the biophase where reception takes place. If two compounds, $A$ and $B$, distribute themselves between, say, water and octanol, they usually do so independently, because the water-A or water-B and the octanol-A or octanol- $\mathrm{B}$ interactions are much stronger than the $\mathrm{A}-\mathrm{B}$ interaction in either phase. Nevertheless, for partition from water into, for example, hexadecane, the previous is not necessarily so because the A-B interaction in the hexadecane (nonpolar) phase may be larger than any hexadecane- $A$ or hexadecane- $B$ interaction. In such cases, the lipophilicity of the A,B mixture will be larger than calculated from the separate values (M. H. Abraham, personal communication, October 1994). This falls into register with the relevance found for the parameter $L^{16}$ (gas-liquid partition coefficient of the irritant VOC on hexadecane at $298 \mathrm{~K}$ ) in our QSAR equation for nasal pungency (Abraham et al., 1996).

In our attempt to balance the mixtures for each subject-weighting the components according to each subject's individual thresholds-we noticed that it was more common for the simpler than for the complex mixtures to have one strongly dominant component. When one component is heavily predominant, it is more difficult to show statistically reliable simple agonism, because the span over which any agonism can be shown becomes so small. Nevertheless, we did not find a significant correlation between the degree of imbalance of the mixtures, measured as root-mean-squared deviation from perfect balance, and degree of agonism.

As already mentioned, we had to rely on previously measured thresholds for one component in Mixture C (1butanol) and for two in Mixture D (1-heptanol and hexyl acetate), and this limits the precision of our results. For single compounds, the ratio between thresholds reported here and in our previous studies averaged 2.5 for pun- gency and 2.2 for odor, with some variation, depending on the particular compound. Insofar as we relied on previously measured thresholds for the three mentioned compounds, our calculation of degree of agonism could be influenced by this source of variation.

Much remains to be investigated regarding human chemosensory thresholds for mixtures of VOCs. One fundamental issue is the study of mixtures where the components vary systematically in their proportions. It is easy to see that such experiments would involve a tremendous amount of work if detailed analytical and sensory measurements - as in this study - are to be undertaken, but the payoff is likely to be substantial in terms of understanding.

The last years have seen an increase in the number of cases where occupants of buildings complain of a wide variety of symptoms that invariably include irritation of mucous membranes (eyes, nose, and throat) as an important component (see Cometto-Muñiz \& Cain, 1992). In most of these cases, chemical analysis of the air shows no individual compound at a high enough level to be responsible for the observed health effects. The present results strongly suggest that the combined action of probably dozens of relatively nonreactive chemicals at levels far below their respective thresholds could precipitate irritation of mucus membranes.

\section{REFERENCES}

Abraham, M. H., Andonian-Haftvan, J., Cometto-Muñiz, J. E., \& CAIN, W. S. (1996). An analysis of nasal irritation thresholds using a new solvation equation. Fundamental \& Applied Toxicology, 31, $71-76$.

AMOORE, J. E. (1986). Effects of chemical exposure on olfaction in humans. In C. S. Barrow (Ed.), Toxicology of the nasal passages, (pp, 155-190). Washington, DC: Hemisphere.

ANHOLT, R. R. H. (1993). Molecular neurobiology of olfaction. Critical Reviews in Neurobiology, 7, 1-22.

BAKER, R. A. (1963). Odor effects of aqueous mixtures of organic chemicals. Journal of the Water Pollution Control Federation, 35 , 728-741.

Berglund, B., \& Olsson, M. J. (1993). Odor-intensity interaction in binary and ternary mixtures. Perception \& Psychophysics, 53, 475 482 .

Brown, K. S., Maclean, C. M., \& Robinette, R. R. (1968). The distribution of the sensitivity to chemical odors in man. Human Biology, 40, 456-472.

Buck, S. H., \& BuRKs, T. F. (1986). The neuropharmacology of capsaicin: Review of some recent observations. Pharmacological Reviews, 38, 179-226.

CAIN, W. S. (1989). Testing olfaction in a clinical setting. Ear, Nose \& Throat Journal, 68, 316-328.

CAIN, W. S., \& Gent, J. F. (1991). Olfactory sensitivity: Reliability, generality, and association with aging. Journal of Experimental Psychology: Human Perception \& Performance, 17, 382-391.

Cain, W. S., Schiet, F. T., Olsson, M. J., \& de Wijk, R. A. (1995). Comparison of models of odor interaction. Chemical Senses, 20, 625-637.

Cometto-Muñiz, J. E., \& CaIn, W. S. (1990). Thresholds for odor and nasal pungency. Physiology \& Behavior, 48, 719-725.

Cometto-Muñiz, J. E., \& CAIN, W. S. (1991). Nasal pungency, odor, and eye irritation thresholds for homologous acetates. Pharmacology, Biochemistry \& Behavior, 39, 983-989.

Cometto-Muñiz, J. E., \& Cain, W. S. (1992). Sensory irritation. Relation to indoor air pollution. In W. G. Tucker et al. (Eds.), Sources 
of indoor air contaminants: Characterizing emissions and health impacts (Annals of the New York Academy of Sciences, Vol. 64l, pp. 137-151). New York: New York Academy of Sciences.

Cometto-Muñ 1 , J. E., \& CAIN, W. S. (1993). Efficacy of volatile organic compounds in evoking nasal pungency and odor. Archives of Environmental Health, 48, 309-314.

Cometto-MuÑız, J. E., \& CAIN, W. S. (1994). Sensory reactions of nasal pungency and odor to volatile organic compounds: The alkylbenzenes. American Industrial Hygiene Association Journal, 55, 811-817.

Cometto-MuÑIZ, J. E., \& CAIN, W. S. (1995a). Olfactory adaptation. In R. L. Doty (Ed.), Handbook of olfaction and gustation (pp. $257-$ 281). New York: Marcel Dekker.

COMETto-MuÑIZ, J. E., \& CAIN, W. S. (1995b). Relative sensitivity of the ocular trigeminal, nasal trigeminal, and olfactory systems to airborne chemicals. Chemical Senses, 20, 191-198.

Cometro-Muñiz, J. E., García-Medina, M. R., \& Calviño, A. M. (1989). Perception of pungent odorants alone and in binary mixtures. Chemical Senses, 14, 163-173.

Cometto-Muñiz, J. E., \& Hernández, S. M. (1990). Odorous and pungent attributes of mixed and unmixed odorants. Perception \& Psychophysics, 47, 391-399.

Geppetti, P., Fusco, B. M., Marabini, S., Maggi, C. A., FanciulLACCI, M., \& SiCUTERI, F. (1988). Secretion, pain and sneezing induced by the application of capsaicin to the nasal mucosa in man. British Journal of Pharmacology, 93, 509-514.

GREEN, B. (1993). Evidence that removal of capsaicin accelerates desensitization on the tongue. Neuroscience Letters, 150, 44-48.

Guadagni, D. G., Buttery, R. G., Okano, S., \& Burr, H. K. (1963). Additive effect of sub-threshold concentrations of some organic compounds associated with food aromas. Nature, 200, 1288-1289.

LASKA, M., \& HUDSON, R. (1991). A comparison of the detection thresholds of odour mixtures and their components. Chemical Senses, 16, $651-662$.

LundBlad, L., LundBerg, J. M., \& ÄNGGÅRD, A. (1984). Local and systemic capsaicin pretreatment inhibits sneezing and the increase in nasal vascular permeability induced by certain chemical irritants. Naunyn-Schmiedeberg's Archives of Pharmacology, 326, 254-261.

Patterson, M. Q., Stevens, J. C., Cain, W. S., \& Cometto-Muñiz, J. E. (1993). Detection thresholds for an olfactory mixture and its three constituent compounds. Chemical Senses, 18, 723-734.
Rosen, A. A., Peter, J. B., \& Middleton, F. M. (1962). Odor thresholds of mixed organic chemicals. Journal of the Water Pollution Control Federation, 35, 7-14.

Sicuteri, F., Fusco, B. M., Marabini, S., Campagnolo, V., Maggi, C. A., Geppetti, P., \& Fanciullacci, M. (1989). Beneficial effect of capsaicin application to the nasal mucosa in cluster headache. Clinical Journal of Pain, 5, 49-53.

SILVER, W. L. (1992). Neural and pharmacological basis for nasal irritation. In W. G. Tucker et al. (Eds.), Sources of indoor air contaminants: Characterizing emissions and health impacts (Annals of the New York Academy of Sciences, Vol. 641, pp. 152-163). New York: New York Academy of Sciences.

Silver, W. L., Farley, L. G., \& Finger, T. E. (1991). The effects of neonatal capsaicin administration on trigeminal nerve chemoreceptors in the rat nasal cavity. Brain Research, 561, 212-216.

Silver, W. L., Mason, J. R., Marshall, D. A., \& Maruniak, J. A. (1985). Rat trigeminal, olfactory and taste responses after capsaicin desensitization. Brain Research, 333, 45-54.

STJÄrne, P., LUndBlad, L., LUNDBERG, J. M., \& ÄNGGÅRd, A. (1989). Capsaicin and nicotine-sensitive afferent neurones and nasal secretion in healthy human volunteers and in patients with vasomotor rhinitis. British Journal of Pharmacology, 96, 693-701.

UNGER, W. G. (1992). Pharmacological and neural bases for eye irritation. In W. G. Tucker et al. (Eds.), Sources of indoor air contaminants: Characterizing emissions and health impacts (Annals of the New York Academy of Sciences, Vol. 641, pp. 176-186). New York: New York Academy of Sciences.

\section{NOTE}

1. A strict application of the terms simple agonism, synergistic agonism, partial agonism, and antagonism would require knowledge of the psychometric function for each individual stimulus and mixture, quite a complex undertaking. In the absence of such functions, our use of the terms in this early investigation overlooks such complexities

(Manuscript received December 27, 1995 revision accepted for publication June 29,1996 .) 